\title{
Characterization of Oryza rufipogon-Derived Resistance to Tungro Disease in Rice
}

\author{
Yuji Shibata, Rogelio C. Cabunagan, Pepito Q. Cabauatan, and Il-Ryong Choi, International Rice Research In-
} stitute, Plant Breeding, Genetics and Biotechnology Division, DAPO Box 7777, Metro Manila, Philippines

\begin{abstract}
Shibata, Y., Cabunagan, R. C., Cabauatan, P. Q., and Choi, I.-R. 2007. Characterization of Oryza rufipogon-derived resistance to tungro disease in rice. Plant Dis. 91:1386-1391.

Rice tungro disease (RTD) is caused by the interaction between Rice tungro spherical virus (RTSV) and Rice tungro bacilliform virus (RTBV), both of which are transmitted by green leafhoppers (GLH). In order to define the resistance against RTD in rice cv. Matatag 9 which was developed by interspecific hybridization between RTD-susceptible cv. IR64 and Oryza rufipogon, the reactions of Matatag 9 to the viruses and GLH were evaluated in comparison with RTDsusceptible and -resistant rice cultivars. The incidences of infection with RTSV and RTBV in Matatag 9 were significantly lower than those in the susceptible parent cv. IR64; however, no substantial differences in virus accumulation were observed between IR64 and Matatag 9 once infected with the viruses. Symptoms in Matatag 9 infected with RTBV and RTSV were milder than those observed in IR64. A higher level of antixenosis to GLH was observed in Matatag 9 compared with IR64. The levels of antibiosis against GLH in Matatag 9 were comparable with those in another GLH-resistant cultivar, and significantly higher than those in RTD-susceptible cultivars. Collectively, these results suggest that tolerance to tungro viruses and resistance to GLH both contribute to the apparent resistance to RTD in Matatag 9, although possible involvement of other resistance mechanisms cannot be excluded.
\end{abstract}

Additional keywords: Nephotettix virescens, Oryza sativa

Rice tungro disease (RTD) is caused by the interaction between Rice tungro bacilliform virus (RTBV) and Rice tungro spherical virus (RTSV) (10). Rice plants infected with RTBV alone exhibit distinctive symptoms such as stunted growth, yellow to yellow-orange discoloration of leaves, and reduced tillering. Although RTSV alone does not cause conspicuous symptoms, except occasional slightly stunted growth, the symptoms become more severe when plants are simultaneously infected with RTBV and RTSV. Both RTSV and RTBV are transmitted in a semipersistent manner by the green leafhoppers (GLH), including Nephotettix virescens $(11,22)$. GLH transmit RTSV and RTBV simultaneously or singly from source plants infected with both viruses. RTSV is independently transmitted by GLH, but RTBV can be transmitted only by GLH which have fed on plants infected with RTSV (11). RTBV is found in both xylem and phloem, whereas RTSV usually is observed only in the phloem tissues of infected plants (23).

Corresponding author: I.-R. Choi

E-mail: ichoi@cgiar.org

Accepted for publication 26 April 2007.

doi:10.1094/PDIS-91-11-1386

This article is in the public domain and not copyrightable. It may be freely reprinted with customary crediting of the source. The American Phytopathological Society, 2007.
RTD is one of the most serious threats to rice production in South and Southeast Asia; therefore, breeding for resistance to RTD is an important component of rice cultivar improvement programs in the region (14). Due to the interdependency among tungro viruses and GLH during disease development, it was assumed that RTD can be controlled by the introduction of host resistance against either of the two viruses or GLH. Initially, rice cultivars resistant to GLH were adopted widely to control RTD (18). However, many of the vector-resistant cultivars sustained severe damage several years after their release because GLH populations were able to adapt to and colonize these cultivars (8). Meanwhile, through the evaluation of more than 40,000 germplasm accessions of cultivated rice (Oryza sativa) for the reactions to tungro viruses, dozens of cultivars were identified to be resistant to RTSV (9). Although some cultivars showed tolerance to RTBV infection, none with a high level of resistance against RTBV were found among the rice cultivars examined $(9,21)$. In order to diversify the sources of resistance against RTD, a wide range of wild Oryza spp. also were examined for their reactions to tungro viruses and GLH. It was reported that few accessions of Oryza spp. such as $O$. rufipogon, $O$. longistaminata, $O$. officinalis, and $O$. ridleyi are tolerant or resistant to tungro viruses, regardless of their levels of resistance to GLH (13,15-17). Particularly, an accession of $O$. rufipogon (International Rice Research
Institute [IRRI] Genetic Resource Center [GRC] accession number 105908) did not show any sign of infection with RTBV or RTSV when artificially inoculated, and it also appeared to be moderately tolerant to resistant to GLH infestation $(13,16)$.

Several traditional rice cultivars and wild Oryza spp. have been used for the improvement of cultivars resistant to RTD. Matatag 9 is a cultivar developed from the cross between a popular indica rice, cv. IR64, and the RTD-resistant accession of O. rufipogon (105908) (13). A high level of resistance in Matatag 9 to RTD was substantiated through field evaluations in areas of high RTD pressure (R. C. Cabunagan and I.-R. Choi, unpublished results). However, the reactions of Matatag 9 to tungro viruses and GLH were inconsistent with those previously reported for the resistant donor O. rufipogon (16). Thus, the mechanism of RTD resistance in Matatag 9 was not well identified and this eventually could become an obstacle for further improvement and sustainable deployment of resistant cultivars from the same donor. In this study, therefore, the levels of resistance in Matatag 9 to the individual biological agents involved in RTD were comparatively assessed in order to phenotypically define Matatag 9 resistance to RTD.

\section{MATERIALS AND METHODS}

Plant material. IR64 (IRRI GRC accession number 66970) is susceptible to RTD and tolerant to GLH (13). Matatag 9 (IR73885-1-4-3-2-1-6) is an RTD-resistant rice cultivar produced by hybridization between $O$. rufipogon and IR64, followed by two backcrosses to IR64 and more than three selfings (13). IR64 and Matatag 9 are similar in their agronomic characteristics (unpublished results). Taichung Native 1 (TN 1) and IR62 were included as susceptible and resistant check cultivars, respectively, in antibiosis (plant traits that adversely affect herbivore performance or survival) tests for GLH (8). Seed of all cultivars were maintained in the Plant Breeding, Genetics, and Biotechnology Division of IRRI.

Insect vector and viruses. The colony of GLH ( $N$. virescens) was maintained on TN 1 (25 to 35 days old) in metal cages. Inocula for RTSV (strain A) and RTBV (common strain) (6) were maintained by successive transmission of viruses to TN 1 . Source plants were tested for the presence of RTSV and RTBV by enzyme-linked immunosorbent assay (ELISA) before 
acquisition feeding access by GLH $(4,7)$. Viruliferous GLH were prepared by allowing a newly emerged adult population to feed on plants infected with RTSV and RTBV for 4 days.

Detection of virus infection. Inoculated plants were evaluated for virus infection by ELISA $(4,7)$. The presence of RTBV or RTSV in leaf extracts was determined by measurement of the absorbance at $405 \mathrm{~nm}$ using an ELISA reader (BioTek Instruments, Winooski). Plants for which the 10fold-diluted extracts exhibited an absorbance value greater than 0.1 were considered infected with tungro viruses. The cutoff absorbance value was determined empirically based on the observation that the absorbance values of 10 -fold-diluted extracts from healthy plants seldom exceed 0.1 , whereas the absorbance values of the extracts from the susceptible control (TN 1) at 21 days after inoculation (DAI) usually exceeded 0.1 .

Susceptibility to virus infection. The test tube inoculation method was used (9) to determine the susceptibility of IR64 and Matatag 9 to tungro viruses. Six-day-old seedlings were inoculated individually with one, three, or five GLH viruliferous for both RTBV and RTSV (RTBV/RTSVviruliferous GLH). The incidence of RTBV (or RTSV) infection represents the percentage of the total number of plants infected, including those infected with RTBV or RTSV alone and those simultaneously infected with both viruses (RTBV/RTSV infection) to the number of plants inoculated. Incidences of infection were determined at 21 DAI based on the results from 40 plants per treatment. The experiment was repeated three times.

Relative quantification of virus accumulation. The levels of tungro virus accumulation in Matatag 9 were compared with those in IR64 to examine whether virus multiplication was affected in Matatag 9. Six-day-old seedlings of IR64 and Matatag 9 were inoculated with three viruliferous GLH per plant by the test tube method. Plants for the evaluation of RTBV accumulation were inoculated with RTBV and RTSV, and those for RTSV accumulation were inoculated with RTSV. Two youngest leaves per seedling were collected from 20 plants of each cultivar at 7 , 14, 21, 28, 35, 42, and 49 DAI. Each plant was used only once for leaf sampling. The optimal dilution rates of leaf extracts for the estimation of virus titer within the proportional range of absorbance values at $405 \mathrm{~nm}$ by ELISA were determined based on the levels of reactivity of serially diluted extracts from TN 1 infected with RTSV and RTBV. Depending on the optimal dilution rate determined, three or four dilutions of leaf extracts between 10- to 320 -fold were made with PBS-T $(0.02 \mathrm{M}$ phosphate buffer [pH 7.4], $0.15 \mathrm{M} \mathrm{NaCl}$, and $0.05 \%$ Tween 20). The diluted extracts were subjected to ELISA. Only samples with absorbance values greater than 0.1 at a 10-fold dilution were used for the estimation of virus accumulation. The absorbance value of a sample at the optimal dilution rate was used for the estimation if the value was within the proportional range. If the absorbance value at the optimal dilution rate was not within the proportional range, the value at another dilution rate that was within the proportional range was used after normalizing it with the ratio of the actual dilution rate to the optimal dilution rate. The normalized values at each time point were averaged to estimate the levels of relative virus accumulation. The experiment was repeated two times.

Host choice test for GLH. Two pots (12 cm in diameter), one planted with five seedlings of IR64 and the other with Matatag 9, were placed $10 \mathrm{~cm}$ apart inside a plastic cage ( 45 by 45 by $45 \mathrm{~cm}$ ) with a mesh window ( 6 by $8 \mathrm{~cm}$ ). Approximately 50 RTBV/RTSV-viruliferous GLH were introduced into the cage when the plants were 10 days old. The number of GLH that alighted on plants in each pot was counted at $0.5,4,8$, and $24 \mathrm{~h}$ after introduction. Insects were removed from the plants at 24 $\mathrm{h}$ after infestation, and the plants were maintained in a greenhouse. Incidences of infection with tungro viruses were evaluated by ELISA at 20 days after the removal of GLH. The experiment was repeated three times. Each experiment was established in a randomized complete block design (RCBD) with 20 replications. The numbers of alighted GLH on Matatag 9 and IR64 were analyzed by a $\chi^{2}$ test to determine whether observed ratios of the numbers of GLH alighted on Matatag 9 to those of IR64 deviated significantly from the ratio of $1: 1$.

Adult GLH longevity. Twenty-six-dayold seedlings of each cultivar were placed individually in test tubes with nylon mesh tops. One newly emerged adult GLH (male or female) was introduced into each tube. Ten seedlings were exposed to GLH of each sex. Seedlings were replaced whenever they showed severe necrosis. The mean longevity for 10 individuals of each sex was determined. The experiment was repeated three times. Each experiment was established in a split-plot design (main effect was cultivar and split plot was sex) with three replications. Data were analyzed by the least significant difference (LSD) test for the means, and by the balanced analysis of variance (BAOV) included in the IRRISTAT package (IRRI, Philippines) to evaluate the significances among cultivars.

Nymph mortality. Six-day-old seedlings were placed individually in test tubes. In all, 10 to 11 second-instar nymphs were introduced into each tube. The number of live nymphs in each tube was counted at $6 \mathrm{~h}$ and 3 days after infestation. The mortality of the nymphs was calculated as the ratio of the GLH number at 3 days after infestation to the initial number at $6 \mathrm{~h}$ after infestation. Ten observations were made for each cultivar per replication. The experiment was repeated three times. Each experiment was established in an RCBD with three replications. Data was analyzed by LSD test and BAOV.

Nymph development. Pregerminated seed were sown individually in $12-\mathrm{cm}$ pots covered with 10-by-60-cm cylindrical mylar cages with fine-mesh nylon screen top and side windows. Five second-instar GLH were introduced into the cage 21 days after sowing. The number of nymphs that developed into adults was recorded daily and the time to reach the adult stage was calculated from the observations. The insect growth index was calculated as the ratio of the percentage in nymphs developing into adults to the mean time from second-instar to adult in days (19). The experiment was repeated three times. Each experiment was established in an RCBD with 20 replications. Data were analyzed using LSD and BAOV.

\section{RESULTS}

Infection with tungro viruses. To minimize the interference by the probable adverse effects of plants on GLH feeding during virus inoculation, viruliferous GLH were allowed access to individual plants placed in tubes for evaluation of resistance to tungro viruses. IR64 and Matatag 9 seedlings were inoculated using one, three, or five GLH to determine differences in virus infection incidences between the cultivars under the increasing inoculation pressure. The incidence of infection with either virus at 21 DAI was significantly higher in IR64 than in Matatag 9 regardless of the number of GLH used for inoculation (Fig. 1). The incidence of RTBV infection in either cultivar when inoculated with three or five GLH was significantly higher than in plants inoculated with one GLH (Fig. 1C). However, no significant differences were observed among the incidences of RTSV infection when plants were inoculated with one to five GLH (Fig. 1B). The incidences of RTBV infection were higher than those for RTSV regardless of the cultivar or number of GLH. Especially, very low incidences of RTSV infection were observed in Matatag 9, with RTSV infection occurring in less than 5\% of the plants even when inoculated with five GLH. The low incidence of RTSV infection resulted in the lower incidence of RTBV/RTSV infection (Fig. 1A) compared with those with RTBV.

Temporal changes in the relative levels of tungro virus accumulation in IR64 and Matatag 9 were compared to evaluate how virus multiplication was affected in Matatag 9. The optimal dilution rates of leaf extract for the estimation of virus titer by ELISA were different among experiments due to the difference in sensitivity among the antibody preparations used in the re- 
spective experiments. The optimal dilution rates were set at 50 - and 80 -fold in the first and second experiments for RTBV, and 20and 80-fold in the first and the second experiments for RTSV, respectively (data not shown). No significant differences were observed between the levels of RTSV accumulation in Matatag 9 and IR64 at the
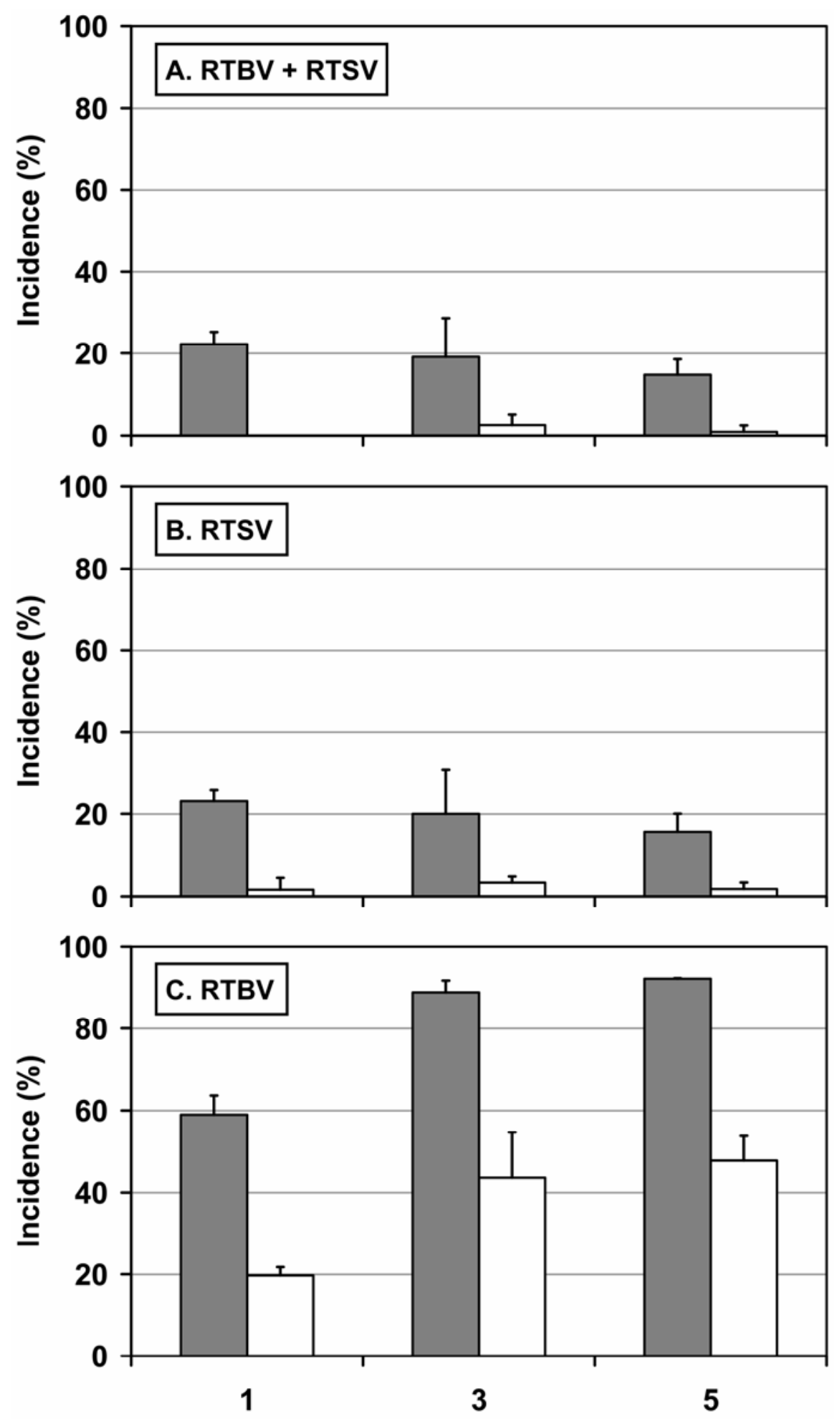

Number of GLH per plant

Fig. 1. Incidences of tungro virus infection in IR64 and Matatag 9 inoculated by the test tube method. The mean incidences for A, Rice tungro bacilliform virus (RTBV) plus Rice tungro spherical virus (RTSV), B, RTSV, and C, RTBV infection in IR64 (gray column) and Matatag 9 (white column) inoculated with one, three, and five RTBV/RTSV-viruliferous green leafhoppers (GLH) per plant were assessed at 21 days after inoculation by enzyme-linked immunosorbent assay. The bars represent the standard deviation of infection incidence. Means and standard deviations were based on three independent experiments.

same sampling dates (Fig. 2C and D). However, the incidence of RTSV infection was consistently lower in Matatag 9 (10 to $50 \%$ ) than in IR64 (55 to 90\%) (data not shown). Likewise, RTBV accumulation was not significantly different between Matatag 9 and IR64 (Fig. 2A and B). The levels of RTBV in both IR64 and Matatag 9 remained nearly in parallel during the period of observation. The incidences of RTBV infection in Matatag 9 (45 to $80 \%$ ) also were lower than those in IR64 (80 to $100 \%$ ) at all sampling dates (data not shown).

Although the levels of RTSV and RTBV accumulation were not different between Matatag 9 and IR64, more severe symptoms developed in IR64 than in Matatag 9 when they were infected with both tungro viruses. Severe stunting and yellowing were observed in IR64 infected with RTBV and RTSV, whereas most plants of Matatag 9 infected with both tungro viruses did not show typical RTD symptoms (data not shown).

GLH preference for IR64 and Matatag 9. The preference of GLH for IR64 and Matatag 9 was assessed by monitoring temporal changes in the numbers of GLH alighted on the two cultivars confined together in one cage. The number of RTBV/RTSV-viruliferous GLH that alighted on IR64 increased up to $8 \mathrm{~h}$ after introduction, and was seemingly maintained at a similar level thereafter (Fig. 3). In contrast, the numbers of GLH on Matatag 9 showed no significant differences among time points. The numbers of GLH alighted on the two cultivars were similar at $0.5 \mathrm{~h}$ after introduction $\left(\chi^{2}=4.90, P=\right.$ 0.24). However, it appeared that GLH alighted on the plants unequally between the two cultivars during the subsequent period of observation $\left(\chi^{2}=48.81,152.33\right.$, and 163.96 at 4,8 , and $24 \mathrm{~h}$ after introduction, respectively; $P<0.01)$.

The plants confined with GLH also were tested for infection with RTBV and RTSV to evaluate the relationship between the numbers of viruliferous GLH alighted on the plants and RTD incidence. The incidences of infection with tungro viruses in Matatag 9 were lower than those in IR64 (Table 1). The incidence of RTSV infection in both cultivars in the host choice test conducted in cages containing 10 plants ( 5 plants each of the two cultivars) and approximately 50 GLH (Table 1) was higher than the RTSV incidence in the individual inoculation test conducted with single plants and five GLH (Fig. 1). Considering the two cultivars collectively, the incidence of RTBV/RTSV infection within the individual pots (five plants per pot) was highly correlated with the numbers of GLH alighted on the plants at $8 \mathrm{~h}$ $(r=0.739, n=120)$ and at $24 \mathrm{~h}(r=$ 0.751 ) after introduction. Under the same assumption, the incidence of RTSV infection also was highly correlated with the 
numbers of GLH alighted on plants at $8 \mathrm{~h}$ $(r=0.744)$ and at $24 \mathrm{~h}(r=0.750)$ after introduction.

Antibiosis in Matatag 9 against GLH. The level of antibiosis to GLH in Matatag 9 was assessed by comparisons of developmental responses of GLH among Matatag 9 and cultivars showing differential reactions to GLH. The longevity of adult GLH on IR64 ranged from 2 to 42 days, and the range on Matatag 9 was from 1 to 28 days. The longevities of both male and female adult GLH were significantly longer on IR64 than on Matatag 9 and IR62 (Table 2). The longevity of adult GLH was longest on TN 1.

Significant differences in the mortality of nymph GLH at 3 days after infestation were observed among the cultivars (Table 2). The mortality of nymph GLH on IR64 was significantly lower than that on Matatag 9, but higher than that on TN 1. The nymph mortality on Matatag 9 was not significantly different from that on IR62.

The nymph development period on Matatag 9 was significantly longer than that on TN 1 and IR64, but was significantly shorter than that on IR62 (Table 2). More than $80 \%$ of nymphs developed into adults on both IR64 and TN 1, whereas less than $60 \%$ of nymphs did on Matatag 9. The higher rates of nymphs to adults and the shorter developmental period in IR64 resulted in a significantly higher growth index for IR64 than for Matatag 9.

\section{DISCUSSION}

Wild Oryza spp. have drawn attention as an alternative genetic resource for favorable traits in breeding of rice cultivars.

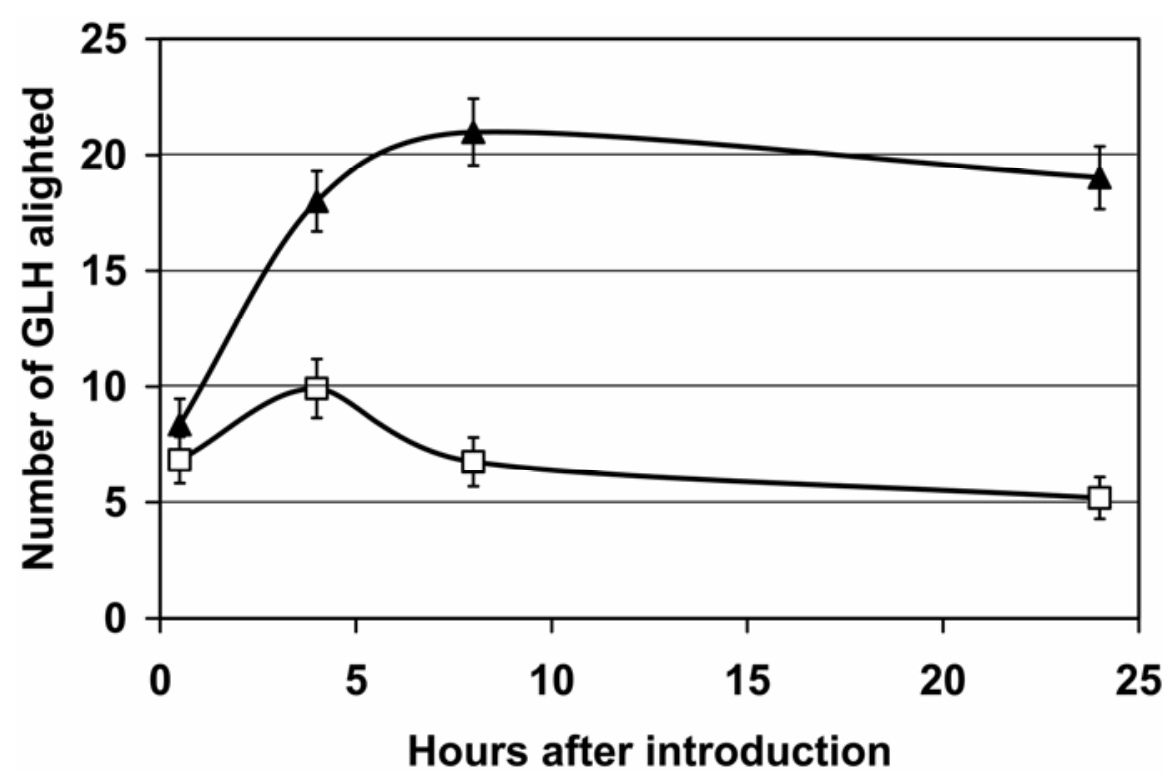

Fig. 3. Temporal changes in the numbers of green leafhoppers (GLH) alighted on IR64 and Matatag 9 during the host choice test. The mean numbers of Rice tungro bacilliform virus and Rice tungro spherical virus (RTBV/RTSV)-viruliferous GLH alighted per five plants of IR64 (black triangles) and Matatag 9 (white squares) are shown. Vertical lines indicate the standard error of mean for the numbers of GLH per five plants at the respective time points. Means and standard error of mean were based on three independent experiments.
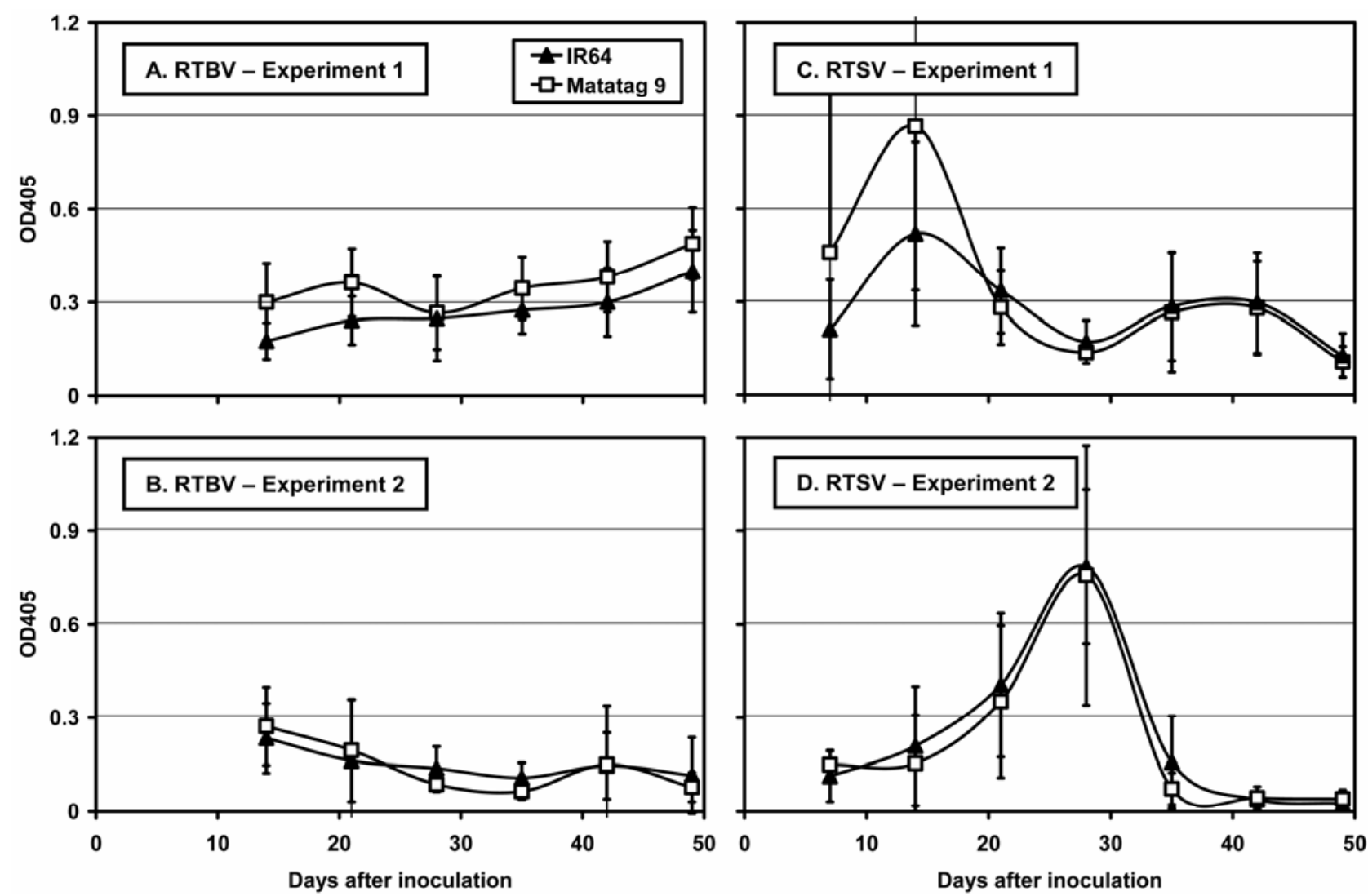

Fig. 2. Temporal changes in the relative levels of tungro virus accumulation in IR64 and Matatag 9 observed in two independent experiments. The relative levels of accumulation for A and B, Rice tungro bacilliform virus (RTBV) and C and D, Rice tungro spherical virus (RTSV) in IR64 (black triangles) and Matatag 9 (white squares) were determined as outlined in Materials and Methods. The error bars represent the standard deviation for normalized absorbance values. Means and standard deviations of the normalized absorbance values were based on the absorbance values from 2 to 20 samples depending on the incidences of virus infection in each cultivar. 
Especially, resistance of wild Oryza spp. to biotic stresses such as rice grassy stunt virus, bacterial blight, blast, and brown leafhopper have been transferred into cultivated rice plants (5). Some accessions of wild Oryza spp. were observed to be tolerant to resistant to tungro viruses and GLH $(13,16,17)$. Enhancement of resistance to RTD in cultivated rice has been achieved through interspecific hybridization with wild Oryza spp. such as O. rufipogon and O. longistaminata. However, the levels of resistance to the individual agents of RTD in the progeny rice plants have not been well evaluated. In this study, phenotypes associated with resistance to RTD in Matatag 9 were compared with those in the recurrent parent IR64, because the two cultivars are similar in agronomic and genetic characteristics except in the reactions to RTD.

It is generally recognized that the levels of virus accumulation in plants change during disease development. Consequently, the difference in the levels of virus accumulation among plants should be examined over a period of a month or longer for more reliable comparison. No significant differences were found in the relative accumulation of RTBV or RTSV between IR64 and Matatag 9 (Fig. 2), yet the symptoms in IR64 were markedly more severe than in Matatag 9. These results suggest that Matatag 9 exhibits tolerance to tungro viruses by suppressing symptom development. It was reported that several traditional cultivars, such as Gam Pai 30-12-15 and Balimau Putih, also exhibited considerably milder symptoms when infected with both tungro viruses (9). Therefore, the apparent tolerance observed in some traditional cultivars and that conferred by $O$. rufipogon may share common genetic features to suppress the development of severe symptoms. However, the low incidence of virus infection in Matatag 9 after GLH inoculation (Fig. 1) indicates that characteristics other than tolerance also may be involved in resistance to tungro viruses in Matatag 9. It is possible that an early event in virus infection, for example, disassembly of virions in plant cells, might be important in reducing infection incidence in Matatag 9, because multiplication and movement of tungro viruses in Matatag 9 were not affected once the infection was established.

Comparison of the levels of antibiosis to GLH among Matatag 9 and other GLHresistant and -susceptible cultivars (Table 2) demonstrated that Matatag 9 is highly resistant to GLH. GLH apparently settled on the plants continuously during the forced inoculation; therefore, the low incidences of tungro virus infection may have resulted from the adverse influence by GLH resistance of Matatag 9 on the feeding behavior of GLH.

Unequal distribution of GLH between Matatag 9 and IR64 in the host choice test (Fig. 3) clearly demonstrated that Matatag 9 is the less-preferred plant. The incidence of RTSV infection was highly associated with the numbers of GLH alighted on the plants at 8 to $24 \mathrm{~h}$ after introduction. The low incidence of RTSV infection associated with low numbers of GLH in Matatag 9 in the host choice test implies that antixenosis (plant traits conferring nonpreference of herbivores) of Matatag 9 may be critical to reducing the incidences of RTSV infection in Matatag 9 under field conditions. Meanwhile, no clear relationship was observed between the incidences of RTBV infection and the numbers of GLH alighted at 8 and $24 \mathrm{~h}$ after introduction in Matatag 9 (data not shown), suggesting that RTBV may have been transmitted into Matatag 9 even during the initial probing of GLH. High incidences of RTBV infection by artificial inoculation also were observed in other GLH-resistant cultivars (12).

The incidences of RTSV infection in both Matatag 9 and IR64 in the host choice test performed in cages containing 10 plants ( 5 plants each of the two cultivars) and approximately 50 GLH (Table 1) were higher than the incidences in the forced inoculation test conducted in the tubes containing a single plant and 5 GLH (Fig. 1B). Therefore, the difference in the incidences of RTSV infection between the two tests suggests that the capability of GLH to transmit RTSV can be affected by the inoculation conditions.

Despite the difficulty in distinguishing resistance to GLH and to tungro viruses, dozens of rice germplasm stocks were identified that exhibit resistance or tolerance to GLH and at least one of the tungro viruses (2). Cosegregation of resistance to GLH and to RTSV also was observed in the progeny of RTD-resistant cv. ARC11554 (20). The results from this study indicate that resistance to GLH and tolerance to tungro virus infections in $O$. rufipogon were transferred together into Matatag 9. Therefore, the apparent resistance to RTD observed in Matatag 9 most probably is due to the combined effects between resistance to GLH and tolerance to tungro viruses, although possible involvement of other mechanisms in the resistance cannot be excluded. The lifespan of resistance to insects in rice may be shortened under continuous monoculture of resistant cultivars (8). In addition, tungro viruses are genotypically and biologically highly variable $(1,3,6)$. Hence, it is highly desirable to regularly monitor the reactions of Matatag 9 with field populations of GLH and tungro viruses for sustainable deployment of Matatag 9.

\section{ACKNOWLEDGMENTS}

This work was partially supported by International Collaborative Research Funds from the Rural Development Administration, Korea. We thank V. Bartolome for her help in statistical analysis, P. Domingo for his assistance in ELISA, and M. Izon for his technical assistance in the maintenance of test plants.

\section{LITERATURE CITED}

1. Arboleda, M., and Azzam, O. 2000. Inter- and intra-site genetic diversity of natural field populations of rice tungro bacilliform virus in the Philippines. Arch. Virol.145:275-289.

2. Azzam, O., and Chancellor, T. C. B. 2002. The biology, epidemiology, and management of rice tungro disease in Asia. Plant Dis. 86:88100.

3. Azzam, O., Yambao, Ma. L. M., Muhsin, M., McNally, K., and Umadhay, K. 2000. Genetic

Table 2. Adult longevity, nymph mortality, and nymph development characteristics of green leafhoppers (Nephotettix virescens) on resistant and susceptible cultivars $^{y}$

\begin{tabular}{|c|c|c|c|c|c|c|}
\hline \multirow[b]{2}{*}{ Cultivar } & \multicolumn{2}{|c|}{ Adult longevity (day) } & \multirow[b]{2}{*}{ Nymph mortality (\%) } & \multicolumn{3}{|c|}{ Nymph development } \\
\hline & Male & Female & & Nymph into adult (\%) & Developmental period (day) & Growth index ${ }^{2}$ \\
\hline IR62 & $8.6 \pm 0.5 \mathrm{a}$ & $9.9 \pm 0.6 \mathrm{a}$ & $66.1 \pm 2.1 \mathrm{c}$ & $62.3 \pm 4.0 \mathrm{~b}$ & $19.6 \pm 0.4 \mathrm{c}$ & $3.2 \pm 0.3 \mathrm{a}$ \\
\hline Matatag 9 & $8.0 \pm 0.5 \mathrm{a}$ & $9.0 \pm 0.5 \mathrm{a}$ & $70.4 \pm 1.7 \mathrm{c}$ & $55.6 \pm 4.3 \mathrm{a}$ & $19.1 \pm 0.4 \mathrm{~b}$ & $3.1 \pm 0.3 \mathrm{a}$ \\
\hline IR64 & $14.3 \pm 0.7 b$ & $15.6 \pm 0.9 \mathrm{~b}$ & $26.6 \pm 1.1 \mathrm{~b}$ & $89.0 \pm 2.5 \mathrm{c}$ & $14.8 \pm 0.3 \mathrm{a}$ & $6.1 \pm 0.2 \mathrm{~b}$ \\
\hline TN 1 & $24.6 \pm 0.5 c$ & $22.7 \pm 0.9 \mathrm{c}$ & $4.0 \pm 0.4 \mathrm{a}$ & $100.0 \pm 0.0 \mathrm{~d}$ & $14.3 \pm 0.3 \mathrm{a}$ & $7.1 \pm 0.2 \mathrm{c}$ \\
\hline
\end{tabular}

y Results are based on three independent experiments. Mean \pm standard error of mean. Values followed by a common letter in a column are not significantly different at the $5 \%$ level by least significant difference test.

${ }^{\mathrm{z}}$ Growth index $=$ percentage of nymphs developing into adult/developmental period. 
diversity of rice tungro spherical virus in tungro-endemic provinces of the Philippines and Indonesia. Arch. Virol. 145:1183-1197.

4. Bajet, N. B., Daquioag, R. D., and Hibino, H. 1985. Enzyme-linked immunosorbent assay to diagnose rice tungro. J. Plant Prot. Trop. 2:1125-129.

5. Brar, D. S., and Khush, G. S. 1997. Alien introgression in rice. Plant Mol. Biol. 35:35-47.

6. Cabauatan, P. Q., Cabunagan, R. C., and Koganezawa, H. 1995. Biological variants of rice tungro viruses in the Philippines. Phytopathology 85:77-81.

7. Cabunagan, R. C., Flores, Z. M., Coloquio, E. C., and Koganezawa, H. 1993. Virus detection in varieties resistant/tolerant to tungro. Int. Rice Res. Notes 18:22-23.

8. Dahal, G., Hibino, H., Cabunagan, R. C., Tiongco, E. R., Flores, Z. M., and Aguiero, V. M. 1990. Changes in cultivar reaction to tungro due to changes in "virulence" of the leafhopper vector. Phytopathology 80:659-665.

9. Hibino, H., Daquioag, R. D., Mesina, E. M., and Aguiero, V. M. 1990. Resistance in rice to tungro-associated viruses. Plant Dis. 74:923926.

10. Hibino, H., Roechan, M., and Sudaresman, S. 1978. Association of two types of virus particles with penyakit habang (tungro disease) of rice in Indonesia. Phytopathology 68:14121416.

11. Hibino, H., Saleh, N., and Roechan, M. 1979. Transmission of two kinds of rice tungro associated viruses by insect vectors. Phytopathology 69:1266-1268.

12. Hibino, H., Tiongco, E. R., Cabunagan, R. C., and Flores, Z. M. 1987. Resistance to rice tungro-associated viruses in rice under experimental and natural conditions. Phytopathology 77:871-875.

13. Khush, G. S., Angeles, E., Virk, P. S., and Brar, D. S. 2004. Breeding rice for resistance to tungro virus at IRRI. SABRAO J. Breed. Gen. 36:101-106.

14. Khush, G. S., and Virmani, S. 1985. Breeding rice for disease resistance. Pages 239-279 in: Progress in Plant Breeding. G. E. Russel, ed. Butterworths, London.

15. Kobayashi, N., and Ikeda, R. 1992. Necrosis caused by rice tungro viruses in Oryza glaberrima and $O$. barthii. Jpn. J. Breed. 42:885-890.

16. Kobayashi, N., Ikeda, R., Domingo, I. T., and Vaughan, D. A. 1993. Resistance to infection of rice tungro viruses and vector resistance in wild species of rice (Oryza spp.). Jpn. J. Breed. 43:377-387.

17. Kobayashi, N., Ikeda, R., and Vaughan, D. A. 1993. Resistance to rice tungro viruses in wild species of rice (Oryza spp.). Jpn. J. Breed. 43:247-255.

18. Razzaque, Q. M., Heinrichs, E. A, and Rapusas, H. R. 1987. Comparative levels of resistance of rice cultivars and weeds against Nephotettix nigropictus (Stal) and Nephotettix virescens (Distant). J. Plant Prot. Trop. 4:75 84.

19. Saxena, K. N., Gandhi, J. R., and Saxena, R. C. 1974. Patterns of relationship between certain leafhoppers and plants. I. Responses to plants. Entomol. Exp. Appl. 17:03-318.

20. Sebastian, L. S., Ikeda, R., Huang, N., Imbe, T., Coffman, W. R., and McCouch, S. R. 1995 Molecular mapping of resistance genes to rice tungro spherical virus and green leafhopper in rice. Phytopathology 86:25-30.

21. Shahjahan, M., Jalant, A. H., Zakri, A. H Imbe, T., and Othman, O. 1990. Inheritance of tolerance to rice tungro bacilliform virus (RTBV) in rice (Oryza sativa L.). Theor. Appl. Genet. 80:513-517.

22. Sogawa, K. 1976. Rice tungro virus and its vectors in tropical Asia. Rev. Plant Prot. Res. 9:21-46.

23. Sta. Cruz, F. C., Koganezawa, H., and Hibino, H. 1993. Comparative cytology of rice tungro viruses in selected rice cultivars. J. Phytopathol. 138:274-282. 